\title{
High-temperature type Ila gratings in 12-ring photonic crystal fibre with germanosilicate core
}

K.cook@usyd.edu.au

A.A.P. Pohl
K. Cook

Interdisciplinary Photonics Laboratories, School of Chemistry, University of Sydney, NSW 1430 Australia

Interdisciplinary Photonics Laboratories, School of Chemistry, University of Sydney, NSW 1430 Australia

Federal University of Technology - Parana, Av. Sete de Setembro, 3165, Curitiba, Brazil, 80.230-901 Interdisciplinary Photonics Laboratories, School of Chemistry, University of Sydney, NSW 1430 Australia

The inscription of efficient Bragg gratings ( $>20 \mathrm{~dB}$ ) in 12-ring photonic crystal fibre with a germanosilicate doped core without the need for hydrogen loading using $193 \mathrm{~nm}$ is demonstrated. We show the characteristic rollover from type I grating to type lla - the first observation of a type lla grating in structured optical fibre. The type Ila grating is shown to survive up to $700^{\circ} \mathrm{C}$ before any degradation in transmission or reflection takes place. [DOI: 10.2971/jeos.2008.08031]

Keywords: Bragg gratings, Photonic crystal fibres

\section{INTRODUCTION}

As the fast-moving field of structured optical fibres continues to evolve, new device and system technologies are emerging that are not viable in conventional optical fibres. Structured fibres, such as photonic crystal fibres (PCFs) [1] and Fresnel fibres [2] offer many advantages over their step-index counterparts. Highly tailorable chromatic dispersion [3], endlessly single mode operation [4], high optical nonlinearities [5] and the ability to permeate the holey cladding with liquids or gases [6] are only some of the qualities that have allowed these fibres to excel as novel fibre lasers and sensors. However, as for conventional fibres, the use of Bragg gratings can significantly extend the functionality of such devices. Due to a range of factors that hinder grating writing, such as scattering by the surrounding lattice [7] and fibre splicing, most grating writing is based on a single photon process in germanosilicate doped cores loaded with hydrogen or deutrium [8]-[11]. The loading of hydrogen itself presents an additional difficulty as the hydrogen can quickly out-diffuse from the core due to the proximity of the cladding holes, even with the inclusion of deuterium [10]. This problem can be avoided by carefully splicing the fibre ends to standard SMF-28 fibre prior to loading in order to seal the holes [8, 9]. Efficient gratings using $248 \mathrm{~nm}$ light have been inscribed by filling of the holes with index matching liquids to overcome the problem of scattering of the transversely-incident writing beam [12]. An alternative approach has been to induce two-photon excitation directly at the $\mathrm{SiO}_{2}$ band edge in fibres with pure silica cores to produce a densification grating [13]. This method eliminates the need for dopants altogether (an attractive quality, since fibre fabrication costs are therefore reduced); however, larger fluencies are required and there is an increased sensitivity to scattering by the structured lattice. Still higher multiphoton processes require liquids inserted into the holes to reduce this scattering [14]. Nevertheless, in many applications, the inclusion of a doped core is necessary in order to achieve sufficient photosensitivity for conventional single photon writing. In this paper, we report on the efficient writing of Bragg gratings in highly nonlinear photonic crystal fibre with a 12-ring holey cladding with no hydrogen loading. A $193 \mathrm{~nm}$ laser source is used to directly excite the germanosilicate core, leading to the formation of a type I and, with continued exposure, a socalled type IIa grating. We also demonstrate the survival of such a grating to temperatures as high as $700^{\circ} \mathrm{C}$ before any degradation occurs.

\section{EXPERIMENTAL SETUP}

The commercially available fibre [15] used for these experiments consists of a triangular core surrounded by 12 rings of holes. A scanning electron micrograph (SEM) of the PCF is shown in Figure 1(a). There is a central core region, diameter $d=(2.1 \pm 0.3) \mu \mathrm{m}$, with a germanium concentration of $\sim 6.7 \mathrm{wt} \%$. Three silica regions define the overall triangular core, combined with a relatively high hole diameter to pitch ratio $(d / \Lambda=0.58)$ generates a flat positive dispersion [16]. This small core size enhances the nonlinearity of this fibre. The high Ge doping of the core ensures the fibre is substantially photosensitive and has been used to write gratings at $248 \mathrm{~nm}$ and $266 \mathrm{~nm}$ with hydrogen [8].

PCF lengths of 10-15 cm were spliced at each end to SMF-28 fibre using a standard arc fusion splicer. The splicing parameters - arc strength, duration and push distance - were carefully selected in order to minimise splice loss while maintaining 

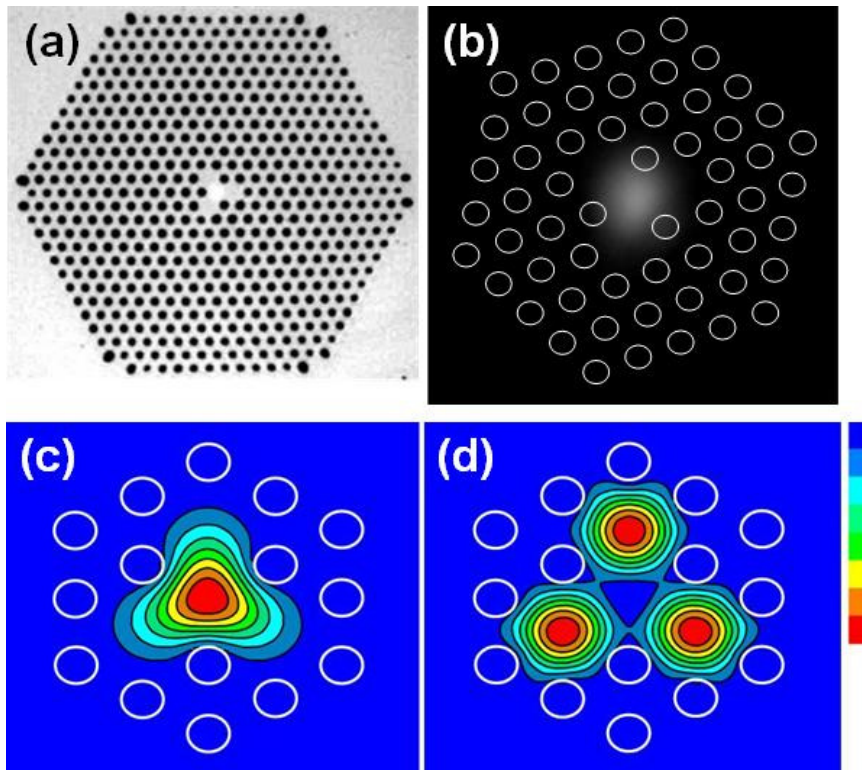

FIG. 1 (a) SEM of highly nonlinear PCF; (b) Imaged mode after splicing; (c) Simulated profile of the fundamental $\mathrm{TEM}_{00}$ mode; (d) Simulated profile of the higher-order TEM $_{01}$ mode.

mechanical durability. The measured loss of $\sim 2 \mathrm{~dB} /$ splice arises mostly from mode mismatch between the two fibres. Alignment during splicing was achieved by coupling $1550 \mathrm{~nm}$ light into the SMF-28 fibre and imaging the output at the end of the PCF using a Vidicon IR camera - this ensured that the fundamental mode was excited when coupling into the PCF. A captured image of the fundamental mode is shown in Figure 1(b); the locations of the first three rings of cladding holes are also shown as a reference. Figure 1(c) shows the calculated fundamental mode and Figure $1(\mathrm{~d})$ shows the $\mathrm{TEM}_{01}$ equivalent higher order triangular mode supported by this fibre. The transmission of the fibre was monitored using an erbiumdoped fibre amplifier (EDFA - 1500-1550 nm) and an optical spectrum analyzer (res. $=0.05 \mathrm{~nm})$. An ArF exciplex (193 nm, $15 \mathrm{~ns}, 10-200 \mathrm{~Hz}$ ) laser was used to inscribe gratings by direct writing through a phase mask $(\Lambda=1063.5 \mathrm{~nm})$. After attenuation and focussing, an energy density up to $500 \mathrm{~mJ} / \mathrm{cm}^{2}$ per pulse at the surface of the fibre was obtained. All gratings were written with a $10 \mathrm{~Hz}$ repetition rate. The attenuation of $193 \mathrm{~nm}$ through the twelve ring layers, along with single photon absorption, ensures two photon contributions are avoided.

\section{RESULTS}

Several gratings were inscribed using various intensities. Figure 2(a) shows the evolution of the grating strength for various intensities. No control of rotation of the optical fibre was used in these experiments which gives rise to $\pm 5 \%$ variation in UV light reaching the core as a result of the scattering and lensing dependence on the rotational orientation of the fibre [7]. No evidence of a multiphoton excitation process is observed and the grating is generated by conventional single photon excitation through germanosilicate core defects at $193 \mathrm{~nm}$
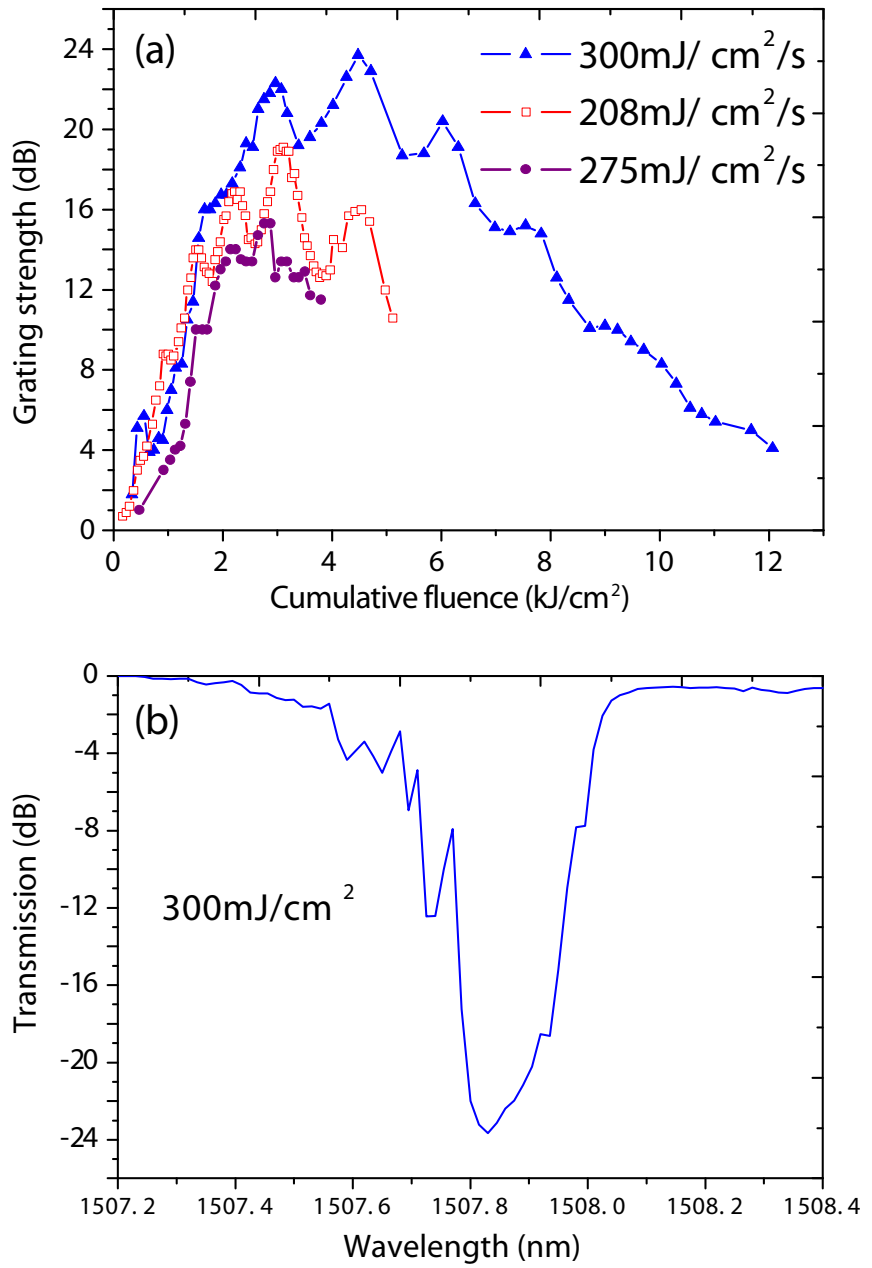

FIG. 2 (a) Change in grating strength with cumulative fluence; (b) Spectrum at the point of $4.5 \mathrm{~kJ} / \mathrm{cm}^{2}$ cumulative fluence for the $300 \mathrm{~mJ} / \mathrm{cm}^{2}$ grating.

The variation in scattering losses at $193 \mathrm{~nm}$ between grating writing accounts for the observation of a stronger grating at $208 \mathrm{~mJ} / \mathrm{cm}^{2}$ than at $275 \mathrm{~mJ} / \mathrm{cm}^{2}$. The grating is strongest at this intensity with an attenuation of around $23 \mathrm{~dB}$. The slight asymmetry of the spectrum is attributed to a small misalignment of the line focus with the fibre. This asymmetry could also arise if there was an unintentional twist in the fibre which would affect the scattering of the light along the fibre length to effectively yield a chirped grating.

Figure 3(a) shows the grating growth curve for a fluence of $500 \mathrm{~mJ} / \mathrm{cm}^{2}$ and an increased cumulative fluence of $50 \mathrm{~kJ} / \mathrm{cm}^{2}$ (writing time of approximately 1.5 hours). The writing was stopped when the grating strength plateaued at $14 \mathrm{~dB}$. From Figure 3(a), a rollover effect is clearly observed at a cumulative fluence of around $20 \mathrm{~kJ} / \mathrm{cm}^{2}$ where there is a reduction in grating strength, or modulation $\Delta n_{\text {mod }}$, consistent with the start of type IIa grating formation [17]-[19]. Type IIa gratings form due to the relief of the anisotropic stresses that build-up during the initial type I grating growth - particularly in the axial and radial directions [20]. These grating types are commonly referred to as negative index gratings $[17,18]$ since the positive change in average index reverses after the erasure of the type I grating. The subsequent change in index modulation is therefore assumed to be negative. 
At lower intensities the rollover effect happens earlier (Figure 2(a)) and the strength of the initial grating is weaker, which is another characteristic of such gratings. Figure 3(a) includes the evolution of the induced refractive index change revealing an initial fast increase followed by an overturn and steady reduction in refractive index. The average index change saturates and is only minimally reversed, the fringe contrast at the rollover point is relatively low with $\Delta n_{\text {mod }} / 2 \Delta n_{a v}=0.08$. The final transmission and normalised reflection spectra are also shown in Figure 3(b) with a grating strength in transmission of around $14 \mathrm{~dB}$ at $1507.78 \mathrm{~nm}$. The transmission spectrum is fairly symmetric with only several weak side lodes which are clearly seen in the spectrum for the normalised reflection (which is on a linear scale). No effort was made to apodise the grating during writing to suppress such side lobes.
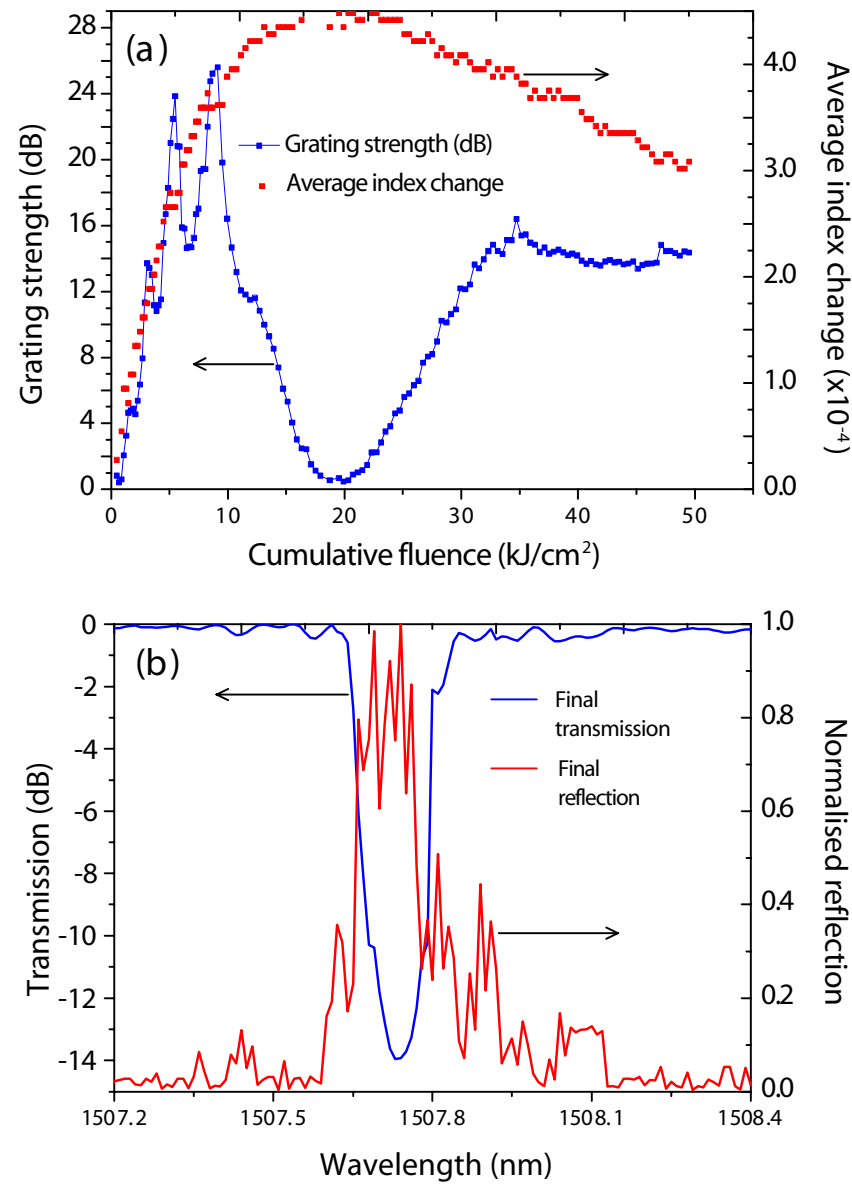

FIG. 3 (a) Grating strength in transmission (blue curve, left hand y-axis) and average index change (red curve, right hand $y$-axis) for a fluence of $500 \mathrm{~mJ} / \mathrm{cm}^{2}$ and an extended UV exposure (approximately 1.5 hours); (b) The corresponding final transmission (blue curve left hand $y$-axis) and normalized reflection (red curve right hand y-axis).

The oscillations observed in all the growth curves for grating strength are attributed to intermodal interference between the fundamental mode and a higher order mode [21, 22] during the writing process. At the time of fibre splicing it was ensured that the PCF guided a single mode in the region of 1500$1550 \mathrm{~nm}$; however, given the attenuation and perhaps lensing induced by scattering it is possible for higher-order modes to be excited, any misalignment and non-uniformity of the UV line focus at the fibre will also contribute. From Figure 3(a) the period of the oscillations is approximately $2 \mathrm{~kJ} / \mathrm{cm}^{2}$. From the index change curve this corresponds to an index difference of $\Delta n \sim 8 \times 10^{-5}$ between the higher order and fundamental mode, which is in fair agreement with the calculated value of $\sim 1 \times 10^{-5}$ using a low resolution simulation of the fibre based on the adjustable boundary conditions Fourier decomposition and finite difference method (ABC-FDM) [23]. As the cumulative fluence increases, these oscillations become weaker and eventually disappear.

Type Ila gratings are known to be highly stable and more resistant to high temperatures than their type I counterparts [17][19]. To investigate the stability of the final grating of Figure 3, the sample was placed in a high temperature oven and subjected to isochronal annealing in steps of $100^{\circ} \mathrm{C}$ up to $800^{\circ} \mathrm{C}$. Above $800^{\circ} \mathrm{C}$, longer dwell durations were used in order to fully observe the grating decay. At a time of 400 minutes, heating at $800^{\circ} \mathrm{C}$ was temporarily halted for an 11 hour (overnight) break - this time period is not included in the graphs for continuity. Figure 4 depicts the overall temperature rise experienced by the grating. The temperature was monitored using a $k$-typed thermocouple and was stable to $\pm 5^{\circ} \mathrm{C}$. The transmission and reflection spectra of the grating were recorded at regular intervals during the heating process.

From Figure 4 it can be seen that the transmission and reflection strengths remain highly stable until the temperature is raised to $700^{\circ} \mathrm{C}$, at which point the grating begins to decay. This decay continues in an exponential fashion when the temperature is raised to $800^{\circ} \mathrm{C}$ and levels-out at around $2.8 \mathrm{~dB}$ and 0.53 (A.U.) for the transmission and reflections strengths respectively. The temperature is then increased to a final value of $900^{\circ} \mathrm{C}$, where the decay is dramatically-accelerated and the grating fully erased after 170 minutes at this temperature (Figure 4 where $t=660$ minutes).

The demonstrated temperature endurance is significantly stronger than conventional type I gratings which tend to anneal-out below $350^{\circ} \mathrm{C}$ [24] and is also greater than the type IIa gratings of $[17,18]$ written in boron-codoped germanosilicate step-index fibres which erase above $500^{\circ} \mathrm{C}$. The decay characteristics are, however, remarkably similar to those observed in [19] where type IIa gratings remain unchanged until $700^{\circ} \mathrm{C}$ - these gratings were also inscribed in boroncodoped germanosilicate step index fibre perhaps fabricated under different conditions to $[17,18]$. The formation of type IIa gratings and their stability with temperature is a complex process that involves many factors such as fibre chemical composition, tension applied during fibre fabrication, tension applied during grating writing and the cumulative fluence used in the process. It is also generally accepted that the decay process of type IIa gratings is complicated by the presence of any residual type I grating, which has been suggested to reduce the maximum temperature that the grating can endure [18]. From our work, the survival of the grating until $700^{\circ} \mathrm{C}$ suggests that the grating is predominantly type IIa and is consistent with [19].

The demonstration of such a stable grating in this highly nonlinear fibre is of profound significance to many applications such as frequency conversion, supercontinum generation and 


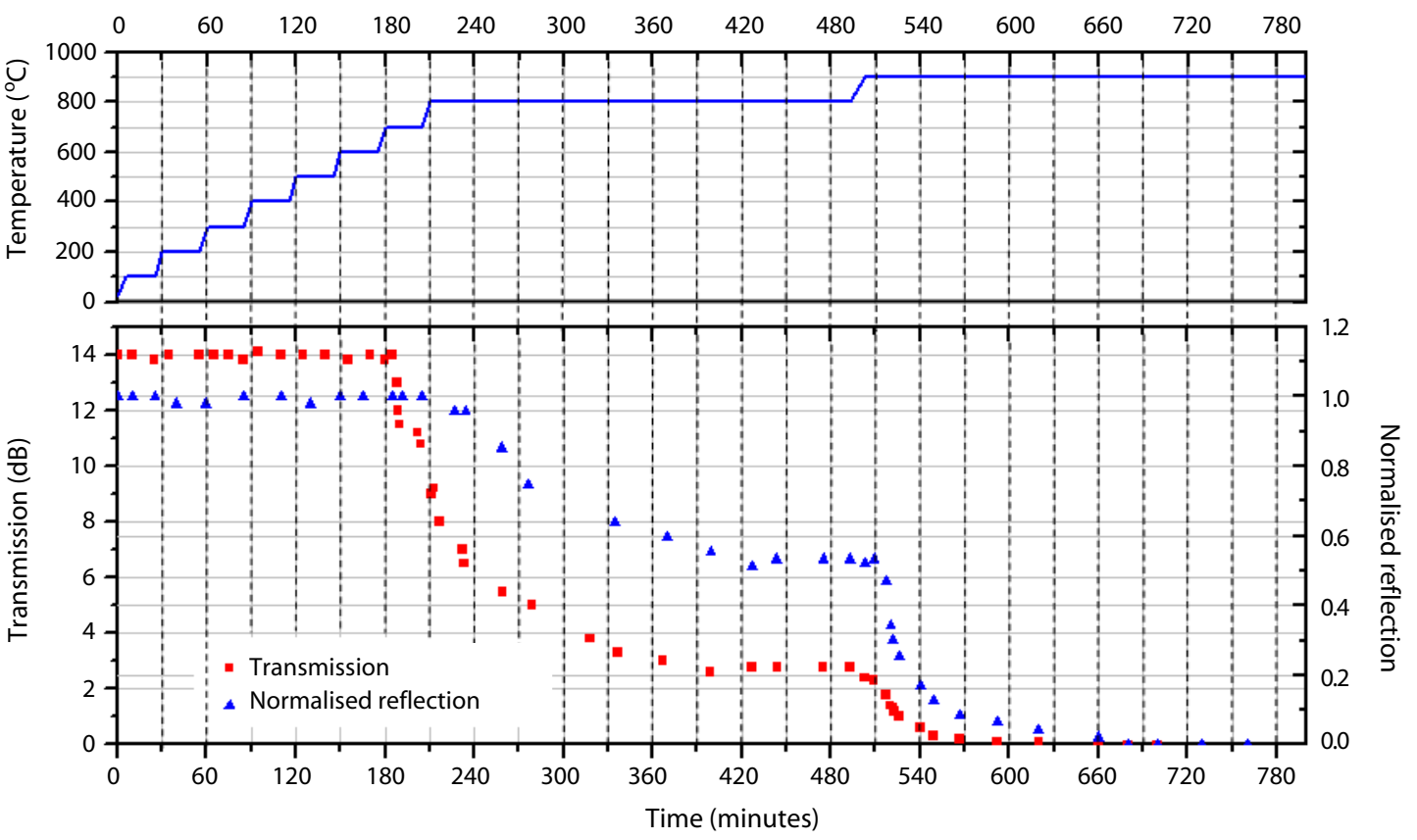

FIG. 4 Temperature rise in $100^{\circ} \mathrm{C}$ steps; grating transmission strength (red curve, left y-axis) and normalized reflection (blue curve, right hand y-axis).

pulse compression - this fibre was specially designed for such applications with a flat dispersion profile and small core diameter. The use of gratings can greatly enhance these nonlinear effects, for example by providing feedback to enhance frequency conversion and also by utilizing the interesting dispersion properties of the actual grating for pulse compression and soliton generation. However, any grating used in these areas has to withstand the high-peak-powers that are usually involved - our durable PCF type IIa grating would be the ideal choice.

\section{CONCLUSION}

In conclusion, we have demonstrated efficient grating writing in PCF with a germanosilicate core and 12 rings of air holes with a large $d / \Lambda=0.58$ using $193 \mathrm{~nm}$. Furthermore, the rollover from a type I grating to a type IIa grating within such a fibre is reported with a final grating strength of up to $14 \mathrm{~dB}$ in transmission. This grating has been shown to endure temperatures up to $700^{\circ} \mathrm{C}$ before the onset of decay, the reflectance is only reduced by around half after a period of 300 minutes at $800^{\circ} \mathrm{C}$ before erasing completely at $900^{\circ} \mathrm{C}$. The performance is superior to early conventional type IIa gratings although similar to more recent work that showed the temperature performance can be enhanced by adjusting the processing conditions. Such a highly stable grating is especially suitable for a variety of applications particularly those involving nonlinear optics.

\section{References}

[1] A. Bjarklev, J. Broeng, and A. S. Bjarklev, Photonic crystal fibres (Kluwer, Academic Publishers, 2003).

[2] J. Canning, "Fresnel Optics Inside Optical Fibres" in Photonics Research Developments, (Nova Science Publishers, 2008).
[3] W. Reeves, J. Knight, P. Russell, and P. Roberts, “Demonstration of ultra-flattened dispersion in photonic crystal fibers" Opt. Express. 10, 609-613 (2002).

[4] T. A. Birks, J. C. Knight, and P. St. J. Russell, “Endlessly single-mode photonic crystal fiber" Opt. Lett. 22, 961-963, (1997).

[5] J. C. Knight, and D. V. Skyrabin, "Nonlinear waveguide optics and photonic crystal fibers” Opt. Express. 15, 15365-15376 (2007).

[6] C. Martelli, J. Canning, D. Stocks, M. J. Crossley, "Water soluable porphyrin in pure silica photonic crystal fiber" Opt. Lett. 31, 21002102 (2006).

[7] J. Holdsworth et al., "Illuminating the core of photonic crystal fiber" presented at the Australian Conference on Optical Fibre Technology, Sydney, Australia, 7-10 July 2008.

[8] H. Sørensen, J. B. Jensen, F. Bruyere, and K. P. Hansen, "Practical hydrogen loading of air silica fibers" presented at Bragg Gratings, Photosensitivity and Poling, Sydney, Australia, 4-9 July 2005.

[9] V. Beugin, L. Bigot, P. Niay, M. Lancry, Y. Quiquempois, M. Douay, C. Mélin, A. Fleureau, S. Lempereur, and L. Gasca, "Efficient Bragg gratings in phosphosilicate and germanosilicate photonic crystal fiber" Appl. Opt. 45, 8186-8193, (2006).

[10] B. J. Eggleton, P. S. Westbrook, R. S. Windeler, S. Spälter, and T.A. Strasser, "Grating resonances in air-silica microstructured optical fibers" Opt. Lett. 24, 1460-1462 (1999).

[11] M. C. Phan Huy, G. Laffont, Y. Frignac, V. Dewynter-Marty, P. Ferdinand, P. Roy, J.-M. Blondy, D. Pagnoux, W. Blanc, and B. Dussardier, "Fiber Bragg grating photowriting in microstructured optical fibers for refractive index measurements" Meas. Sci. Technol. 17, 992-997, (2006).

[12] H. Sørensen, J. Canning, J. Lægsgaard, K. Hansen, and P. Varming, "Liquid filling of photonic crystal fibers for grating writing" 0 pt. Commun. 270, 207-210 (2007).

[13] N. Groothoff, J. Canning, E. Buckley, K. Lyttikainen, and J. Zagari, "Bragg gratings in air-silica structured fibers" Opt. Lett. 4, 233-235 (2003).

[14] N. Groothoff, J. Canning, N. Jovanovic, G. D. Marshall, and M. J. Withford, "Gratings in large diameter air-clad optical fibre using a 
femtosecond laser" presented at Bragg Gratings, Photosensitivity and Poling, Quebec City, Canada, 2-6 September 2007.

[15] http://www.crystal-fibre.com/datasheets/NL-1550-POS-1.pdf

[16] K. P. Hansen, "Dispersion flattened hybrid core nonlinear photonic crystal fiber" Opt. Express 11, 1503-1509 (2003).

[17] L. Dong, W. F. Liu, and L. Reekie, "Negative index gratings formed by a 193-nm excimer laser" Opt. Lett. 21, 2032-2034 (1996).

[18] L. Dong, and W. F. Liu, "Thermal decay of fiber Bragg gratings of positive and negative index changes formed at $193 \mathrm{~nm}$ in a boroncodoped germanosilicate fiber" Appl. Opt. 36, 8222-8226 (1997).

[19] N. Groothoff, and J. Canning, "Enhanced type IIA gratings for hightemperature operation" Opt. Lett. 29, 2360-2362 (2004).

[20] M. Sceats, and J. Canning, Proceedings of Summer School on Photosensitivity in Optical Waveguides and Glasses, H.G. Limberger, ed., (Ecole Polytechnique Federale Lausanne, Lausanne, Switzerland, 1998).

[21] J. Canning, and A. L. C. Carter, "Modal interferometer in situ measurements of induced core index change in optical fibers" 0 pt. Lett. 22, 561-563 (1997).

[22] D. Káèik, I. Turek, I. Martinèek, J. Canning, N. A. Issa, and K. Lyytikäinen, "Intermodal interference in a photonic crystal fibre" Opt. Express 12, 3465-3470 (2004).

[23] N. A. Issam, and L. Poladian, "Vector wave expansion method for leaky modes of microstructured optical fibres" J. Lightwave Technol. 21, 1005-12 (2003).

[24] S. R. Baker, H. N. Rourke, V. Baker, and D. Coodchild, "Thermal decay of Bragg gratings written in boron and germanium codoped silica fiber" J. Lightwave Technol. 15, 1470 (1997). 